\title{
Long term outcome after subarachnoid haemorrhage of unknown aetiology
}

\author{
Liisa M Pyysalo, ${ }^{1}$ Tero T Niskakangas, ${ }^{1}$ Leo H Keski-Nisula, ${ }^{2}$ Veikko J Kähärä, ${ }^{2}$ \\ Juha E Öhman ${ }^{1}$
}

${ }^{1}$ Tampere University Hospital, Department of Neurosurgery, Tampere, Finland

${ }^{2}$ Medical Imaging Centre, Tampere, Finland

\section{Correspondence to}

Dr L M Pyysalo, Tampere University Hospital, Department of Neurosurgery, P 0 Box 2000 Tampere FIN-33521, Finland; liisa.pyysalo@uta.fi

Received 30 December 2010 Revised 21 February 2011 Accepted 15 March 2011 Published Online First 6 April 2011

\begin{abstract}
Background and purpose The aim of this study was to assess the long term outcome after non-aneurysmal subarachnoid haemorrhage (SAH).

Methods 1154 patients with SAH were treated in our hospital between 1989 and 1999. From this patient population, 97 patients had a non-aneurysmal SAH. All hospital records and death certificates were studied and 33 patients were examined by MRI and MR angiography more than 9 years (mean 12 years) after the initial bleeding.
\end{abstract}

Results The cohort consisted of 97 patients. Mean follow-up time was 9 years (range 0-19). During the follow-up period, 13 patients (13\%) died. Four (4\%) died from the initial bleeding less than 5 weeks after the initial haemorrhage. There was no delayed mortality due to SAH or subsequent bleedings. MR angiography revealed no new findings in 33 surviving patients.

Conclusions Excess mortality during the first year after SAH was higher than $4 \%$, and remained thereafter comparable with the general population. There were no rebleedings and MR imaging did not reveal any vascular pathology that could explain the earlier SAH.

\section{INTRODUCTION}

On average, in $15 \%(5-30 \%)$ of patients with spontaneous subarachnoid haemorrhage (SAH), no obvious source of bleeding can be demonstrated, even with high quality four vessel cerebral digital subtraction angiography (DSA). ${ }^{1-8}$ The cause of angiogram negative SAH has not been established but a venous or capillary source, ruptured perforating artery, low flow vascular malformation and short segment arterial dissection have all been postulated. $^{9-11}$ Some researchers do not accept this and speculate that spontaneous thrombosis, vasospasm, destruction of the aneurysm by haemorrhage, narrowing of the aneurysmal neck, alterations in blood flow, inadequate angiographic technique or observer error may be responsible. ${ }^{2}$ Outcome of angiogram negative $\mathrm{SAH}$ is good in $90 \%$ of cases. ${ }^{4} 781213$ However, many groups report non-specific symptoms such as headache, neuropsychological deficits or depressive symptoms in up to $62 \%$ of patients. ${ }^{14-16}$ The rebleeding rate is approximately $5 \%(0-10 \%)^{78} 15-18$ and the risk of death in non-aneurysmal bleeding has been estimated to be $0-15 \% 24781213151718$ Greebe and Rinkel ${ }^{15}$ concluded that patients with an angiographic negative perimesencephalic pattern of $\mathrm{SAH}$ have a normal life expectancy but Hawkins et al ${ }^{17}$ found the cumulative proportional survival after 22 years to be $69 \%$ compared with an expected survival of $89 \%$ in patients with SAH of unknown aetiology.

To the best of our knowledge there are no studies analysing intracranial arterial status very long after initial bleeding or that have reported the incidence of de novo aneurysms in patients having SAH of unknown aetiology. The aim of this study was to assess long term outcome after non-aneurysmal SAH.

\section{METHODS \\ Patients}

Our hospital serves as a primary and secondary care centre for patients with SAH, with a catchment area of more than 1 million. Between 1989 and 1999, a total of 1154 patients with symptoms and signs of spontaneous, non-traumatic SAH were admitted to our hospital. Of these, 97 (8.4\%) patients were included in the study because the aetiology of the SAH could not be verified by repeated four vessel cerebral angiography or postmortem examination. SAH was diagnosed by CT in $80(82 \%)$ cases and by a blood positive lumbar puncture in $17(18 \%)$ cases. The diagnosis 'SAH NOS' was based on DSA in 96 patients and postmortem examination in one patient. All hospital records were studied and the latest outcome of patients was evaluated from the most recent notes in the hospital records or by telephone interview. Clinical status at the time of the bleeding was assessed according to the Hunt and Hess scale. ${ }^{19}$ Glasgow Outcome Scale (GOS) ${ }^{20}$ was used to evaluate clinical status at the time of discharge and at follow-up visits. Primary CT images were re-evaluated to assess Fisher grades and the presence of hydrocephalus. All angiograms were reviewed independently by a neuroradiologist, neurointerventionalist and neurosurgeon. Thereafter, the results were reviewed and a consensus statement for each study was produced. Surviving patients resident in our hospital catchment area were contacted, offered an MRI examination and interviewed to assess clinical outcome and possible rebleeding episodes. A total of 33 were studied with $M R I$ and $M R$ angiography (MRA), and interviewed at our outpatient clinic. Follow-up MRI and MRA were compared with the findings of earlier studies. Surviving patients not studied with MRI lived outside our hospital catchment area, did not answer our letter, were unwilling to participate in the study or had contraindications to MRI. Two surviving patients were lost to clinical follow-up. Thirteen of 97 patients died, and death certificates were obtained from Statistics Finland. The study was approved by the Hospital Ethics Committee and Statistics Finland. 


\section{MRI protocol}

The follow-up MR imaging was performed with the $1.5 \mathrm{~T}$ unit (GE Signa HD, Milwaukee, USA) with one channel head coil. MRA was supplemented with cross sectional imaging, including fluid attenuation inversion recovery, and T1 and T2* sequences, mainly to identify ischaemic parenchyma, ascertain the size of the CSF spaces and persisting blood degradation deposits. The imaging parameters for the non-contrast three-dimensional time of flight angiography were: TR 30, TE 2.5 , FOV $22 \times 16.5 \mathrm{~cm}$, slice thickness $1.0 \mathrm{~mm} /$ interpolated to $0.5 \mathrm{~mm}$, matrix $320 \times 224$. Magnetisation transfer contrast and flow compensation were included.

\section{Statistical analysis}

Statistical analysis was performed using NCSS (NCSS, Kaysville, Utah, USA) statistical software. Categorical variables were compared using the Fisher exact two tailed test. The level of significance was set at $p<0.05$. Excess mortality of the patients was measured by one relative survival ratio. The relative survival ratio is the ratio of the actuarial observed survival rate divided by expected survival rate of a comparable group of patients from the Finnish general population matched by sex, age and calendar time. Relative survival measures the survival experience of the patients corrected for competing risks of death. Relative survival ratios were estimated by survival package SURV3 V.3.01 (Finnish Cancer Registry, Helsinki, Finland).

\section{RESULTS}

Patients were aged 21-76years (mean 52) at the time of bleeding. There were 35 men and 62 women. Most patients had a good clinical status on admission (table 1). In the deceased group, patients had a poorer clinical status and higher Fisher grades on primary CT compared with the surviving group. There

Table 1 Population characteristics

\begin{tabular}{lll}
\hline & Surviving group $(\mathbf{n}=\mathbf{8 4})$ & Deceased group $(\mathbf{n = 1 3})$ \\
\hline Mean age (years) & 52 & 53 \\
Sex & & \\
Male & 31 & 4 \\
$\quad$ Female & 53 & 9 \\
Diagnosis & & \\
CT & 67 & 13 \\
Lumbar puncture & 17 & 0 \\
Fisher & & \\
1 & 16 & 0 \\
2 & 17 & 4 \\
3 & 20 & 5 \\
4 & 23 & 4 \\
Hydrocephalus & 30 & 4 \\
Hunt and Hess & & \\
I & 40 & 2 \\
II & 32 & 2 \\
III & 10 & 5 \\
IV & 1 & 3 \\
V & 1 & 1 \\
GOS at discharge & & \\
I & 0 & 2 \\
II & 0 & 1 \\
III & 10 & 7 \\
IV & 41 & 2 \\
V & 33 &
\end{tabular}

GOS, Glasgow Outcome Score. was no other statistically significant difference between the surviving and deceased groups.

Thirteen of 97 patients (13\%) died during the 868 patient-year follow-up period. Four (4\%) died from the initial bleeding of the non-aneurysmal SAH: two immediately and two patients in 1 month after a poor clinical state. All four patients had severe bleeding on the initial CT (Fisher 3 or 4, non-perimesencephalic type of bleeding). Three patients died of cardiovascular diseases, three of malignancies and three due to traumatic causes. Mortality in the first year after bleeding was more than $4 \%$ in excess of that expected in a comparable group from the general population, both among women and men. Thereafter, mortality of the patients was close to the level in the general population. There were no rebleedings. Ninety-four per cent of the surviving group (79/84) had recovered well (GOS 4 or 5 ) and the reason for the lower GOS was related to SAH in only two $(2 \%)$ patients (figure 1). There was no difference in outcome between patients studied with and without MRI.

Thirty-three patients were studied with MRI. Mean follow-up time was 12 years (range 9-18). No new aneurysms were detected in MRA images or any other vascular pathology that could explain the previous bleeding. Six patients $(18 \%)$ had infarctions in MRI. Two were already seen during the treatment period after clinically and radiologically proven vasospasm. Four infarctions were not detected in earlier images and only two of those four patients had been treated for clinical stroke. Leukoaraiosis was seen in 11 (34\%) patients. Signs of superficial haemosiderin were present in two patients $(6 \%)$.

\section{DISCUSSION}

After SAH, initial angiographic findings are negative for a bleeding source in approximately $15 \%$ of patients. $^{1-8} \mathrm{~A}$ traditional concept states that angiogram negative SAH carries a more benign prognosis than aneurysmal SAH. However, some patients experience morbidity and mortality because of recurrent haemorrhage or vasospasm. ${ }^{14}{ }^{21-23}$ Repeat DSA within 2 weeks after the bleeding is commonly performed. Although repeat DSA provides identification of the source of bleeding in up to $20 \%$ of cases, the source remains undetected in the majority of patients. ${ }^{22-24}$ The incidence of angiogram negative $\mathrm{SAH}$ was $8.4 \%$ in our series and this concurs with recent series. $^{1-8}$

We found no new arterial vasculopathies or other aetiologies for previous SAH in those 33 patients studied. It is a weakness of our study that we performed MRI in only $34 \%$ of patients. However, those surviving patients we could not reach had a poorer clinical status and we believe that this selection bias is unlikely to influence the MRI findings. Infarctions were detected in six (18\%) patients, which is significantly less than in patients with aneurysmal SAH. ${ }^{25-27}$ Two infarctions were caused by vasospasm and

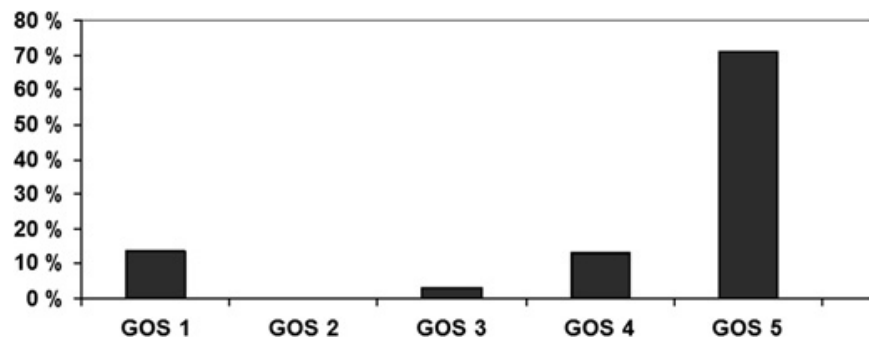

Figure 1 Present Glasgow Outcome Score (GOS) of patients with subarachnoid haemorrhage of unknown aetiology. 
two were incidental findings. Leukoaraiosis was seen in 11 (34\%) patients and this was less than in aneurysmal SAH patients and not more than the prevalence in a neurologically health age cohort. $^{25-28}$ Earlier studies of angiogram negative SAH have reported a subsequent bleeding rate of up to $10 \%$. 8 17 1829 However, the latest reports have detected no rebleedings and neither did we. ${ }^{15} 16$

The outcome is much better in SAH patients with unknown aetiology than in aneurysmal SAH patients. ${ }^{2} 4781213151718$ Eighty-one per cent of patients had recovered well (GOS 4 or 5 ) and poor outcome was related to SAH in only six (6\%) patients in our series. The risk of death from non-aneurysmal bleeding has been estimated to be $0-15 \%$. $^{2} 4781213151718$ In our series, $4 \%$ of patients died of angiogram negative SAH. Overall mortality in our series was $13 \%$ compared with $47 \%$ mortality in aneurysmal SAH patients in our hospital at the same time. ${ }^{30}$ In the long term outcome study, patients with SAH of unknown cause had reduced life expectancy compared with expected numbers. ${ }^{17}$ However, in that study, as many as $22 \%$ of all SAH patients had no cause on angiogram, which was not repeated, raising the suspicion that aneurysms were missed. Greebe and Rinkel, ${ }^{15}$ however, found no difference in mortality between the general population and patients with a perimesencephalic pattern of non-aneurysmal SAH. This study was, however, involved only a subgroup of all patients with SAH of unknown aetiology and to the best of our knowledge our study is the only one in the modern imaging modality era to assess the long term outcome of patients with SAH of unknown aetiology.

\section{CONCLUSION}

Excess mortality during the first year after SAH was higher than $4 \%$, and remained thereafter comparable with the general population. There were no rebleedings and MR imaging did not reveal any vascular pathology that could explain the previous $\mathrm{SAH}$. Based on our results, long term follow-up is not needed, and after appropriate angiographic studies, patients should be informed about the benign nature of this rare type of SAH.

Acknowledgements The authors would like to thank Tapio Luostarinen, MSc, of the Finnish Cancer Registry for help and advice in statistical problems.

Funding The study was financially supported by EVO-funding of Pirkanmaa Health District and Maire Taponen Foundation.

Competing interests None.

Ethics approval The study was conducted with the approval of the Tampere University Hospital Ethics Committee.

Provenance and peer review Not commissioned; externally peer reviewed.

\section{REFERENCES}

1. Broderic JP, Brott TG, Duldner JE, et al. Initial and recurrent bleeding are the major causes of death following subarachnoid hemorrhage. Stroke 1994;25:1342-7.

2. McMahon J, Dorsch N. Subarachnoid haemorrhage of unknown aetiology: what next? Crit Rev Neurosurg 1999;9:147-55.
3. Claassen J, Carhuapoma JR, Kreiter KT, et al. Global cerebral edema after subarachnoid hemorrhage: frequency, predictors, and impact on outcome. Stroke 2002:33:1225-32.

4. Andaluz N, Zuccarello M. Yield of further diagnostic work-up of cryptogenic subarachnoid hemorrhage based on bleeding patterns on computed tomographic scans. Neurosurgery 2008:62:1040-6.

5. Pobereskin LH. Incidence and outcome of subarachnoid haemorrhage: a retrospective population based study. J Neurol Neurosurg Psychiatry 2001;70:340-3.

6. Schaller C, Raueiser B, Rohde V, et al. Cerebral vasospasm after subarachnoid haemorrhage of unknown aetiology: a clinical and transcranial Doppler study. Acta Neurochir (Wien) 1996;138:560-8.

7. Kawamura S, Yasui N. Clinical and long-term follow-up study in patients with spontaneous subarachnoideal haemorrhage of unknown aetiology. Acta Neurochir (Wien) 1990;106:110-14

8. Gómez PA, Lobato RD, Rivas JJ, et al. Subarachnoid haemorrhage of unknown aetiology. Acta Neurochir (Wien) 1989;101:35-41.

9. Van der Schaaf IC, Velthuis BK, Gouw A, et al. Venous drainage in perimesencephalic hemorrhage. Stroke 2004;35:1614-18.

10. Mathews MS, Brown D, Brant-Zawadzki M. Perimesencephalic nonaneurysmal hemorrhage associated with vein of Galen stenosis. Neurology 2008;70:2410-11.

11. Küker W, Thiex R, Block F. Hyperacute perimesencephalic subarachnoid hemorrhage: demonstration of blood extravasation with MRI. J Comput Assist Tomogr 1999;23:521-3.

12. Lang EW, Khodair $\mathrm{A}$, Barth $\mathrm{H}$, et al. Subarachnoid hemorrhage of unknown origin and the basilar artery configuration. J Clin Neurosci 2003;10:74-8.

13. Rinkel GJ, Wijdicks EF, Vermeulen $\mathrm{M}$, et al. Outcome in perimesencephalic (non-aneurysmal) subarachnoid hemorrhage: a follow-up study in 37 patients. Neurology 1990;40:1130-2.

14. Marquardt G, Niebauer T, Schick U, et al. Long term follow up after perimesencephalic subarachnoid haemorrhage. J Neurol Neurosurg Psychiatry 2000;69:127-30

15. Greebe $\mathbf{P}$, Rinkel GJ. Life expectancy after perimesencephalic subarachnoid hemorrhage. Stroke 2007;38:1222-4.

16. Madureira S, Canhão P, Guerreiro M, et al. Cognitive and emotional consequences of perimesencephalic subarachnoid hemorrhage. J Neurol 2000;247:862-7.

17. Hawkins TD, Sims C, Hanka R. Subarachnoid haemorrhage of unknown cause: a long term follow-up. J Neurol Neurosurg Psychiatry 1989;52:230-5.

18. Jain VK, Hedge $T$, Easwaran RK, et al. Benign subarachnoid haemorrhage (subarachnoid haemorrhage of unknown aetiology). Acta Neurochir (Wien) 1987:86:89-92.

19. Hunt WE, Hess RM. Surgical risks as related to time of intervention in the repair of intracranial aneurysms. J Neurosurg 1968;28:14-20.

20. Jennett B, Bond M. Assessment of outcome after severe brain damage. Lancet 1975;1:480-4.

21. Franz G, Brenneis C, Kampfl A, et al. Prognostic value of intraventricular blood in perimesencephalic nonaneurysmal subarachnoid hemorrhage. J Comput Assist Tomogr 2001;25:742-6.

22. Schwartz TH, Solomon RA. Perimesencephalic nonaneurysmal subarachnoid hemorrhage: review of the literature. Neurosurgery 1996;39:433-40.

23. Tatter SB, Crowell RM, Ogilvy CS. Aneurysmal and microaneurysma "angiogram-negative" subarachnoid hemorrhage. Neurosurgery 1995;37:48-55.

24. van Gijn J, Rinkel GJ. Subarachnoid haemorrhage: diagnosis, causes and management. Brain 2001;124:249-78.

25. Bendel $\mathbf{P}$, Koivisto $T$, Könönen $\mathbf{M}$, et al. MR imaging of the brain 1 year after aneurysmal subarachnoid hemorrhage: randomized study comparing surgical with endovascular treatment. Radiology 2008;246:543-52.

26. Kivisaari RP, Salonen 0, Servo A, et al. MR imaging after aneurysmal subarachnoid hemorrhage and surgery: a long-term follow-up study. AJNR Am J Neuroradiol 2001;22:1143-8

27. Pyysalo L, Keski-Nisula L, Niskakangas T, et al. Long-term MRI findings of patients with embolized cerebral aneurysms. Acta Radiol 2011;52:204-10.

28. Salonen 0, Autti T, Raininko R, et al. MRI of the brain in neurologically healthy middle-aged and elderly individuals. Neuroradiology 1997;39:537-45.

29. Ruelle A, Lasio G, Boccardo M, et al. Long-term prognosis of subarachnoid hemorrhages of unknown etiology. J Neurol 1985;232:277-9.

30. Pyysalo LM, Keski-Nisula LH, Niskakangas TT, et al. Long-term follow-up study of endovascularly treated intracranial aneurysms. Interv Neuroradiol 2010;16:361-8. 